eISSN: 2659-6482

DOI: https://doi.org/10.14201/pmrt.26502

\title{
VICTORIA HAZAN Y SUS GRABACIONES DE MÚSICA SEFARDÍ EN NY (1942)
}

\section{Victoria Hazan and his Recordings of Sephardic Music in NY (1942)}

Christos KANELLOS MALAMAS

(Universidad de Salamanca)

guitobo@hotmail.com

RESUMEN: No parece ser muy habitual encontrarse con un repertorio musical turco cantado en español, incorporado en las fiestas y reuniones en los «Café Aman» de los barrios de Manhattan, Brooklyn y Bronx de Nueva York (NY) la primera mitad de siglo XX. Canciones que forman parte de la vida de las tabernas y narran historias humanas acompañando así las danzas y las comidas. Un repertorio que sigue las formas musicales de la tradición musical otomana y el sistema modal turco. Las voces cantantes, inmigrantes de distintos lugares de Asia Menor, Grecia, Armenia, Siria, traen consigo un legado musical, un repertorio que han aprendido de nacimiento, como parte de su cultura y lo trasladan a los barrios de NY creando, de esa manera, el mismo ambiente cultural que vivían en las tabernas de Estambul y Esmirna por la misma necesidad de expresión, comunicación y desahogo.

Una de las voces más brillantes que han quedado inmortalizadas, gracias a las grabaciones de Metropolitan y Kaliphon, es la de Victoria Hazan, de origen sefardí, exiliada de Esmirna, que ha dejado un legado de veintiséis canciones, entre ellas diez en ladino. En el presente trabajo nos acercamos a este repertorio para proponer un análisis que nos permite entender esas canciones a través del sistema musical del makam, así como clasificarlo en modos, entender las formas que la cantante usa en las grabaciones y finalmente poder explorar la posibilidad de reproducirlo y usarlo como cante vivo en un repertorio de concierto usando instrumentos apropiados. 


\title{
Palabras clave: Victoria Hazan; música sefardí; Café Aman.
}

\begin{abstract}
It does not seem to be very common to find a Turkish musical repertoire sung in Spanish, incorporated in the parties and meetings in the "Café Aman» of the Manhattan, Brooklyn and Bronx neighborhoods of NY during the first half of the 20th century. Songs that are part of the life of the taverns and narrate human stories thus accompanying the dances and meals. A repertoire that follows the musical forms of the Ottoman musical tradition and the Turkish modal system. The singing voices, immigrants from different places in Asia Minor, Greece, Armenia, Syria, bring with them a musical legacy, a repertoire that they have learned from birth, as part of their culture and they transfer it to the neighborhoods of NY creating with that way the same cultural environment that they lived in the taverns of Istanbul and Izmir for the same need for expression, communication and relief. One of the most brilliant voices that have been immortalized, thanks to the Metropolitan and Kaliphon recordings, is that of Victoria Hazan, of Sephardic origin, exiled from Smyrna, who has left a legacy of twenty-six songs, including ten in Ladino. In the present work we approach this repertoire to propose an analysis that allows us to understand these songs through the makam musical system, as well as classify it into modes, understand the forms that the singer uses in the recordings and finally be able to explore the possibility of reproduce it and use it as live cante in a concert repertoire using appropriate instruments.
\end{abstract}

Keywords: Victoria Hazan; Sephardic music; Café Aman.

\section{INTRODUCCIÓN}

La inmigración de la población de Asia Menor, Armenia y Siria a principios de siglo XX a NY ${ }^{1}$ fue la causa de la creación de barrios populares en Manhattan, Brooklyn y Bronx, donde cada una de las distintas nacionalidades desarrollaba, de alguna manera, la vida que habían dejado atrás en su tierra natal. Uno de los puntos de encuentro fue la taberna, como de costumbre, donde la gente tenía la oportunidad de encontrarse. Armenios, sirios, griegos, turcos y judíos bebían,

1 Rifat, Bali N. (2013). From Anatolia to the New World Life Stories of the First Turkish Immigrants to America (p. 63). Estambul. Aparecen entre todas las poblaciones desde 1900 hasta 1923 más de 300.000 personas en NY. 
cantaban, bailaban y comían juntos tal como lo hacían en los barrios de ciudades como Esmirna y Estambul.

En estos lugares de encuentro ${ }^{2}$ de diferentes culturas, se expresaban en un entorno popular, alegre, de gastronomía, música y baile, las distintas tradiciones musicales donde los músicos tocaban los instrumentos de su propia tradición y los cantantes cantaban en diversos idiomas y estilos musicales. Más que un lugar concreto, era una situación donde se permitía la expresión artística del pueblo sin ningún prejuicio o miedo. Esa mezcla se refleja, y a la vez es la causa de ella, en las canciones que se cantaban en las tabernas y queda también grabada en los trabajos discográficos que en el presente trabajo analizamos, como es el caso de Victoria Hazan.

Una de las lenguas que encontramos en el repertorio musical dentro de ese contexto es la que se denomina ladino, la lengua que hablan los sefardíes. Es curioso el hecho de encontrar música y melodías que pertenecen a un sistema modal oriental, muy desarrollado melódicamente hablando, pero cantado en español. Es muy frecuente también encontrar las mismas melodías que las cantantes cantaban en las tabernas en el repertorio tradicional turco o griego.

Muchas son las preguntas que surgen: ¿cómo adaptaban estos textos a las melodías preexistentes?, ¿había un repertorio de melodías populares que se usaban como una especie de trampolín para los textos?, ¿se improvisaban los textos?, ¿qué función tenía la música sefardí en ese puzle de culturas y músicas?

\section{ALGUNOS DATOS BIOGRÁFICOS}

Son pocos los datos que tenemos acerca de esa gran cantante ${ }^{3}$. Victoria Hazan nació el 15 de abril de 1896 en Salihli de Izmir (Esmirna). Sus padres eran Moshe y Rivka Ninio. Nació en una familia de cantores, amantes de la música tradicional turca, de hecho, no solo destacaba por su voz, sino también por el instrumento que tocaba.

Alrededor de 1920 se traslada en Nueva York tras la expulsión de los judíos de la costa de Asia Menor. En la sinagoga cantaba y tocaba el ud; en las tabernas cantaba en turco, griego, ladino, armenio y hebreo. Llego a ser presidenta de la United Sisterhood Benevolent Society (Bronx). Tras la muerte de su primer marido sus amigos músicos la convencieron para que entrara en el estudio de grabación y

2 En los barrios de Estambul, estos lugares se llamaban «teke» y es el mismo lugar que los griegos llaman «Café Aman» donde se reunían para cantar y bailar.

3 Sobre todo de fuentes en línea como: http://sephardicmusic.org/artists/Hazan,Victoria/Hazan,Victoria.htm, https://canaryrecords.tumblr.com/post/190578978378/quilted-flowers-1940s-albanian-epirot 
finalmente a partir de 1940 graba con Metropolitan y Kaliphon 12 canciones en turco, 10 en ladino, 2 en griego. Murió el 21 de noviembre de 1995 en Nueva York.

\section{LAS 10 CANCIONES SEFARDÍES GRABADAS EN 1940 CON METROPOLITAN}

Victoria Hazan nos dejó grabadas diez canciones cantadas en ladino, un pequeño repertorio de la tradición musical sefardí, pero con una gran riqueza a nivel melódico y melismático. En ese repertorio encontramos tres distintas formas musicales que provienen de la música turca. Es relevante el hecho de que entre las diez canciones grabadas encontremos nueve en diferentes modos, algo que indica el nivel interpretativo y la capacidad vocal que tenía Hazan.

- En primer lugar, la forma $s a r k \imath^{4}$, muy común en el repertorio popular de la música turca. Repertorio ligero, cantado en las tabernas, muchas veces asociado a danzas, con fraseo menos desarrollado que el del repertorio del palacio ${ }^{5}$, normalmente con estribillo. A pesar de su corta duración, en los sarkı, se llega desarrollar lo que es el modo 6 . La temática de las letras de este repertorio suele ser profana. En esta forma encontramos las siguientes seis canciones: «El Cante por la Victoria», «Me Kemi y me Enflami», «No Riygas con mi», «Seda amarilla son tus Cavellos», «Un Anio Ay», «Un Día Yo Bizi».

- En segundo lugar, la forma gazel es la forma de improvisación vocal por excelencia de la música turca donde el cantante va improvisando con letras inventadas en el momento desarrollando ampliamente el modo en todo su ámbito sonoro. Los intermedios entre estrofas suelen ser improvisaciones leves también por parte de los instrumentistas. El gazel es una de las formas más difíciles de interpretar porque requiere primero una flexibilidad vocal muy elevada por la realización de melismas rápidos descendentes $\mathrm{y}$ ascendentes y segundo un conocimiento de los distintos modos muy amplio para poder realizar las modulaciones ${ }^{7}$, aparte del registro que debe

4 Significa «canción».

5 A diferencia de las grandes composiciones de la música del palacio (Pesrev) donde encontramos piezas de muy larga duración, el sarki tiene aproximadamente una duración de 3-4 minutos no tanto por el número de las estrofas, sino por el desarrollo melódico: frases rápidas. Algo parecido al estilo semineumático del canto gregoriano.

6 Como explicamos más adelante nos referimos al desarrollo melódico en todo el ámbito sonoro de la escala, lo cual crea las distintas modulaciones y cambios de centros tonales o de gravedad.

7 Modulación en el sentido literal de la palabra: paso de un modo a otro. 
tener el cantor para realizarlo. En esta forma encontramos dos canciones: «Mis Penserios» y «No Ay Luz».

- Encontramos también en ese repertorio tan reducido una tercera forma de libre interpretación, más bien adaptación del texto a una melodía, preexistente en el repertorio popular turco. En esta peculiar forma encontramos 2 piezas: «Lagrimas Vertere» $\mathrm{y}$ «Todas mis Esperansas», que son en realidad dos sarkı del repertorio turco, pero que Hazan adapta, de forma libre, el texto en ladino a estas melodías. Es muy interesante observar cómo la cantante usa las notas importantes de la melodía para realizar las cadencias adaptando las palabras a ella.

\section{EL SISTEMA MODAL ${ }^{8}$}

Toda la música grabada por Victoria Hazan se adapta al sistema musical que se denomina makam ${ }^{9}$, ampliamente desarrollado en el Imperio otomano. Es un sistema no temperado, sin elementos de polifonía, que tiene un amplio desarrollo melódico con una riqueza muy grande en cuando a ornamentación. La música que se usa está asociada a un mismo repertorio independientemente de la lengua cantada, algo muy común de las grabaciones de la época ${ }^{10}$. Para comprender ese repertorio en su contexto musical nos haría falta familiarizarnos con ciertos aspectos fundamentales como el sistema de los modos y su funcionamiento, la división del tono en varias partes ${ }^{11}$, las principales características de los modos, el sentido de cadencia, entre otros.

El sistema modal turco, como cualquier música melódica sin textura polifónica, requiere para su funcionamiento ciertas características que a la vez son las principales herramientas para su desarrollo. Estas son:

8 Existe mucha bibliografía sobre la teoría de la música turca sobre todo en turco, griego e inglés. Para mayor comodidad citamos un interesante tratado en inglés: Signell, L. Karl (1977). Makam, modal practice in Turkish art music. Asian Music Publications

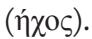

9 Otras denominaciones que indican lo mismo: sistema modal, modo, modalidad, echos

10 Véase también en línea: https://canaryrecords.tumblr.com/post/190578978378/quilted-flowers-1940s-albanian-epirot, donde se encuentran cantantes de distintas nacionalidades cantando en diferentes idiomas, pero siempre dentro del contexto musical del makam, como, por ejemplo: Amalia Vaka, Rosa Eskenazy, cantantes griegas que también cantaban en turco, griego y hebreo.

11 El sistema musical turco divide el tono en 9 partes-comas. En realidad, es una adaptación de la teoría musical griega antigua que considera el sistema perfecto el de las dos octavas, $\delta \imath \varsigma \delta \imath \alpha \pi \alpha \sigma \omega ́ v$, y clasifica los géneros según dónde se encuentra el semitono dentro de la célula principal que se usaba para la construcción de las escalas que es el tetracordo. 
1. Escala

2. Notas importantes

3. Cadencias

4. Desarrollo o discurso melódico

\section{Escala}

La escala es el ámbito sonoro que establece el orden entre las notas con las que se va a desarrollar la melodía. En el lenguaje del makam existen y se usan más de 130 diferentes escalas que nacen de la combinación de los distintos tetracordos hacia lo agudo y hacia los graves. Las escalas se clasifican en géneros según los tetracordos usados como base. Por ejemplo, entre las canciones analizadas, como vamos a ver, encontramos nueve diferentes escalas.

\section{Notas importantes}

Las notas importantes o centros de gravedad son los sonidos a los cuales, a lo largo del discurso melódico, se da más importancia que otros. Eso, a la vez, determina tanto el movimiento melódico como la intención a la hora de la improvisación y la afinación en la interpretación ${ }^{12}$. Dichos centros de gravedad se convierten en centros importantes. Esa es la razón por la que muchas veces confundimos ciertas notas a la hora de la transcripción y las caracterizamos como inestables. En realidad, no son inestables, sino que se justifican por el discurso melódico.

\section{Cadencias}

Las cadencias son los descansos en las notas importantes que determinan a la vez el final de la frase. Se producen tanto en el medio del discurso melódico como al final y casi siempre coinciden con la nota base.

\section{Discurso melódico}

Es la característica más importante del makam. Es, en realidad, la manera de abordar todo lo anterior y también determina si el modo es ascendente o descendente, es decir, si su desarrollo melódico va hacia lo grave o hacia lo agudo o ambos casos. Normalmente, en una pieza de larga duración se desarrolla todo el ámbito

12 Cuando se da importancia a una nota en la escala, en la mayoría de las veces, ciertas notas alrededor de ella (aunque hay ciertas excepciones) cambian su estado natural (tal y como están en la escala original) y se modifican momentáneamente para amoldarse al nuevo centro de gravedad. Se trata de una especie de modulación melódica momentánea, ya que al volver al modo original dichos cambios se anulan. 
sonoro del modo ${ }^{13}$. Ese tipo de composiciones (Pesrev) se dividen en varias partes y cada una de ellas desarrolla un ámbito distinto. Si el desarrollo melódico del modo es descendente la primera parte se desarrolla en la región grave del modo. Las partes siguientes van desarrollando poco a poco las regiones más agudas con las modulaciones que son posibles y finalmente aparece la vuelta hacia el ámbito sonoro inicial ${ }^{14}$.

Cuando las piezas son como las que estamos tratando, es decir, repertorio de taberna de corta duración, el desarrollo es más limitado y se hace más concentrado adaptándose al fraseo melódico más reducido. La pieza «No Riyas con mi» es un buen ejemplo de dicho tipo de desarrollo.

Es importante tener constancia del sistema de escritura utilizado en el lenguaje musical turco a la hora de la transcripción de las piezas, lo cual coincide en parte con el sistema convencional del pentagrama, pero respecto al uso de las alteraciones es diferente. La teoría del makam turco divide el tono en nueve partes-comas, y de alguna forma hay que representar estas divisiones en el papel. Para eso, se usan los signos de alteración que se conocen (sostenido y bemol), pero también se añaden algunos más, para indicar la cantidad de comas que hay que subir o bajar según la escala. Eso, a la vez, hace que la lectura sea difícil para un músico no versado en la materia. Por otro lado es lo más cercano y apropiado para la transcripción de las piezas ${ }^{15}$.

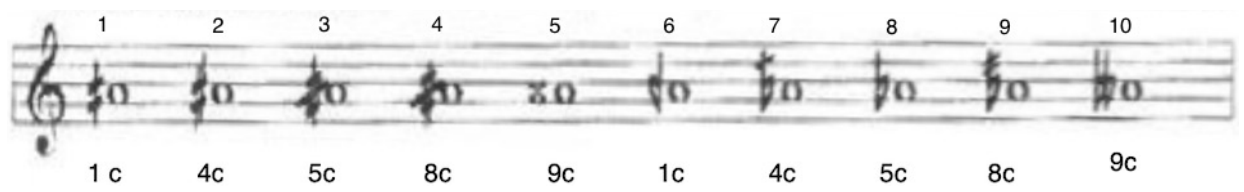

Figura 1. Los signos actuales de alteración de la música turca.

Como podemos observar (Fig. 1), de la necesidad de representar las diferentes escalas surge la creación de distintos símbolos, pero a la hora de la interpretación esto no se cumple. En realidad, estos signos tienen un valor aproximado y tenemos

13 Dicho ámbito depende de la utilización de células de tetracordos más agudas o más graves de la escala base del modo.

14 Las modulaciones posibles que cada makam desarrolla están asociadas al repertorio que nos ha llegado hasta ahora. El estudio comparativo entre las piezas de un mismo makam dan un resultado común no solo en cuanto a las modulaciones, sino a todos los elementos del desarrollo del makam.

15 Existe también la notación bizantina que en nuestra opinión es más adecuada para la interpretación de estas músicas, pero es una notación de uso bastante reducido ya que se usa básicamente en Grecia y algunas comunidades de Armenia, Siria, Líbano y Rumania. 
que tratarlos teniendo en cuenta la flexibilidad vocal del cantor que los interpreta. Si escuchamos los audios de Victoria Hazan, observamos que los intervalos cambian según el movimiento melódico ascendente o descendente, según si se da énfasis a una nota importante y eso determina de forma absoluta las distancias entre las notas. Si quisiéramos transcribir de forma fiel lo que escuchamos en el audio de cualquiera de las piezas analizadas, deberíamos usar muchos más signos que los anteriores en la partitura, dado que las mismas notas cambian constantemente su estado según, como ya hemos dicho, su movimiento melódico o, mejor dicho, su dirección.

La solución para la transcripción es comprender el funcionamiento del sistema del makam para considerar correctamente el papel que juegan las notas en su contexto y su dirección.

Otro problema que nos encontramos en este tipo de repertorio es la ornamentación. Por lo general, las músicas de tradición oriental tienen un grado elevado de melismas y notas añadidas, ligaduras, portamentos, escalas rápidas direccionales, trinos, vibratos de notas reales y otros matices personales de cada cantor. En concreto, en la música turca, estos elementos juegan un papel protagonista. Nos enfrentamos a una cantante de muy alto nivel vocal interpretativo, con una flexibilidad y manejo de la modalidad impresionante. Tenemos una cantidad alta de información detrás de la línea melódica principal que en realidad es muy sencilla, en la mayoría de los casos. La pregunta surge cuando queremos reflejar todos los matices en el papel. ¿Cómo lo hacemos?, y, también, ¿ tiene sentido hacerlo? En las transcripciones que presentamos aquí, no hemos incluido las ornamentaciones porque consideramos que es un trabajo independiente al presente ya que hay muchas variaciones de ornamentos para los mismos giros melódicos y requiere una atención y una clasificación especial.

Aunque la armadura de cada modo nos indica las notas que están alteradas, tenemos que tener en cuenta que se trata de un valor aproximado y no real, ligado más al gusto del intérprete que incluso a la propia tradición musical en sí. Eso a la vez crea problemas a la hora de la interpretación. Digamos que no es un repertorio que fácilmente puede interpretar cualquiera que lee música por lo que hemos comentado anteriormente, pues la cantidad de información que hay detrás de la partitura es muy grande, tanto a nivel melódico y melismático como interválico.

Ese tipo de situaciones, aparentemente incomodas, debidas a la forma de transmisión de estas músicas ${ }^{16}$ crea muchas veces distintos tipos de conflictos a

16 El aprendizaje de las músicas de tradición oral se basa principalmente en la memoria. El fraseo melódico, sobre todo en las manos de cantores de alta capacidad vocal, está a su libre elección en el momento de la ejecución. Lo mismo con la ornamentación, siendo una información adicional, la cual no se piensa a la hora de la interpretación de la melodía, también está a la libre elección del intérprete. Muestra clara de eso son las distintas líneas melódicas que 
la hora del análisis y principalmente de la transcripción e interpretación. Sobre todo, porque en una misma nota se pueden encontrar cuatro valores distintos.

A continuación, presentamos las canciones con un análisis musical y estructural básico y también incluimos dos transcripciones propias. Como nota base de la escala diatónica hemos escogido el $\mathrm{do}^{17}$, que lo asociamos con la nota rast por mayor comodidad en la lectura aunque la altura de los sonidos en la interpretación es relativa, depende de la tesitura que cada uno tiene y donde cada cantor se siente más cómodo para cantar. Las notas correspondientes en toda la escala serían: do-Rast, re-Dugah, mi-Segah, fa-Cargah, sol-Neva, la-Huseuni, sib-Acem, si-Evic, do (octava)-Gerdaniye, re (octava)-Muhayer.

Las diferentes escalas usadas en las canciones son las siguientes ${ }^{18}$ :

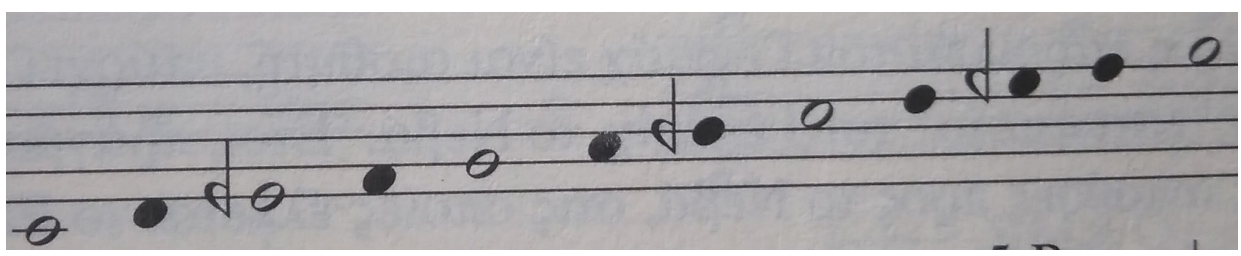

Figura 2. Escala diatónica.

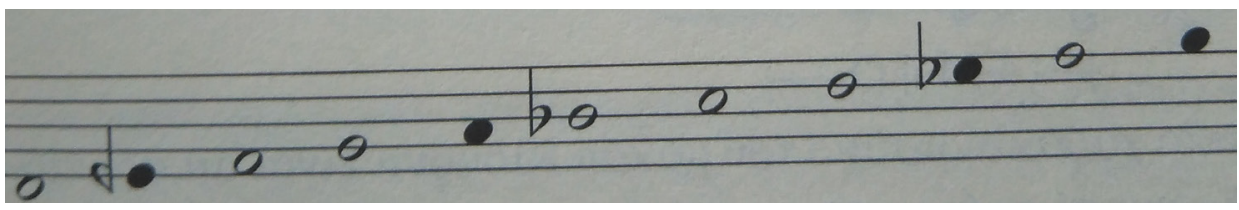

Figura 3. Escala diatónica con el sexto rebajado, acem.

cada cantante emplea sobre una misma dirección melódica. En nuestro caso podemos observar cómo Hazan adapta las palabras a melodías y ornamentos espontáneos en las dos canciones con melodía preexistente: «Lagrimas Vertere» y «Todas mis Esperanzas».

17 Aunque el sistema musical turco ha establecido como base del Rast la nota sol porque sigue la teoría modal griega del sistema perfecto de las dos octavas donde se desarrollan todos los tetracordos.

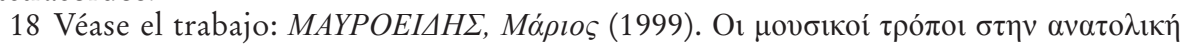
$\mu \varepsilon \sigma o ́ \gamma \varepsilon 10 . A \theta \dot{\eta} v \alpha$ : Fagotto. 


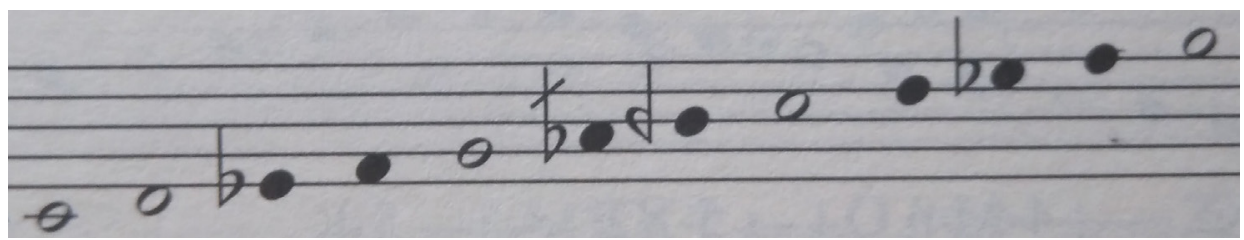

Figura 4. Escala diatónica-cromática del makam nibavent.

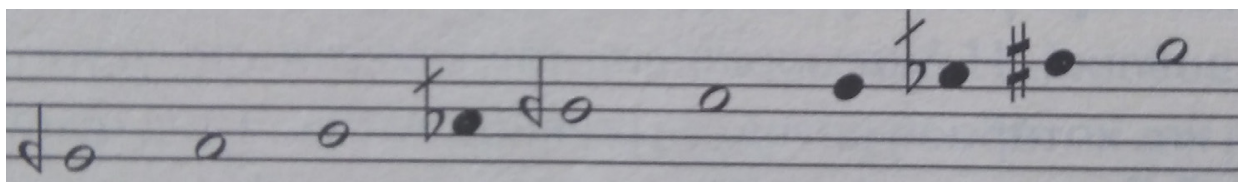

Figura 5. Escala cromática suave.

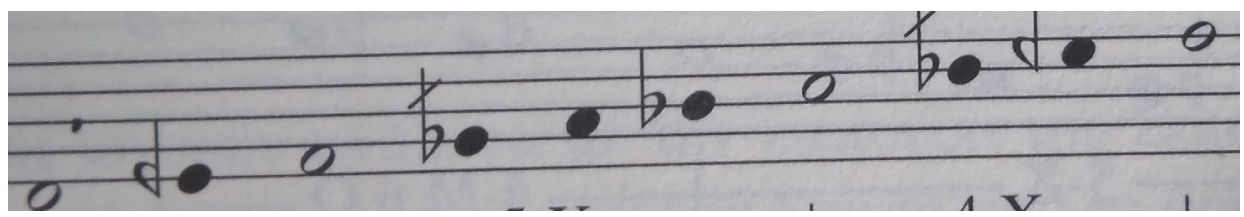

Figura 6. Escala diatónica-cromática del makam sabah.

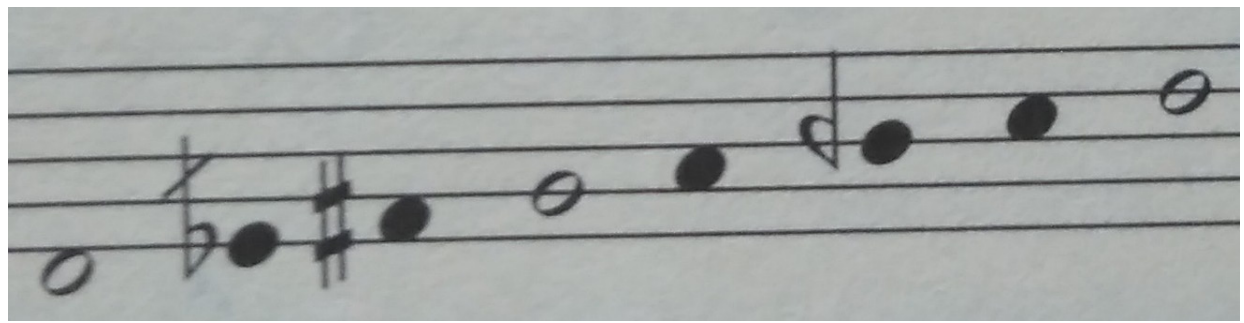

Figura 7. Escala cromática.

Ediciones Universidad de Salamanca / 묘 Popular Music Research Today, 3, 2 (2021), pp. 41-67 


\title{
5. PRESENTACIÓN DE LAS CANCIONES
}

\section{1. «Me Kemi y me Inflami»}

Número: $3001-\mathrm{A}^{19}$

Artista: Hazan, Victoria

Otros artistas: letras de Victoria Hazan. Música de Nick D(oneff), violín. Oriental Trío (Doneff; Melko Melkon, oud; Kanuni Garbis [Bakirgian], kanun).

Matrix: C.V.-3001-A

Grabado: 1942 en NY

\author{
Análisis musical \\ Makam: buseyni \\ Escala: diatónica ${ }^{20}$ \\ Base: dugah \\ Notas importantes: dugah, buseyni
}

Idiomatismos sonoros: en primer lugar hay que destacar que el segundo y el sexto grado (segah, evic) de por sí son dos sonidos no temperados y se encuentran un poco más rebajados en la escala ${ }^{21}$. Por eso en esta escala, en la armadura, estos dos sonidos se encuentran con el bemol al revés ${ }^{22}$.

Aun así, en movimientos melódicos descendentes hacía el primer grado, el segundo grado (segah) se encuentra aún más rebajado para dar más énfasis a esa cadencia hacía dugah.

Otro comportamiento característico de este makam es el comportamiento del sexto grado (evic). En melodías que se desarrollan en el tetracordo agudo del modo (buseyni-mubayer), el quinto grado (buseyni) se convierte en el nuevo centro de gravedad que a su vez determina la altura del sexto (evic) y en htm

19 Datos en línea: https://www.sephardicmusic.org/artists/Hazan,Victoria/Hazan,Victoria.

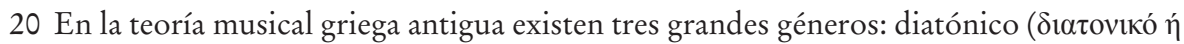

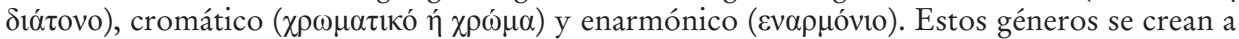
raíz del lugar donde se encuentra el semitono dentro del tetracordo. Posteriormente, en el canto bizantino se mantienen los nombres de los géneros indicando tres grandes familias de modos, pero la función del semitono desaparece. En el género diatónico están los modos que sus escalas están construidas a través de los tres tonos bases $(\delta i \alpha / \tau$ tóvos): tono mayor, tono menor y tono mínimo, que coinciden con la segunda mayor temperada, otra segunda 1-3 comas rebajada y una ultima 2-4 comas rebajada. La teoría musical turca reproduce estos principios.

21 Es decir que la segunda dugah-segab no es la del sistema temperado (1 tono) sino un poco más baja y la sexta dugab- evic no es la sexta mayor sino un poco más baja.

22 Véase la Figura 1. 
consecuencia ese último sigue usando la alteración n. ${ }^{\circ} 6$ (Fig. 1). De modo contrario, cuando la melodía se mueve hacia el tetracordo grave con la intención melódica hacia el dugah, el sexto grado se rebaja bastante más con el «b» (alteración n. ${ }^{\circ} 8$ Fig. 1$)^{23}$.

Desarrollo: la entrada en este makam se suele realizar en la nota buseyni y a lo largo del desarrollo el discurso melódico va bajando, enfatizando grados en el tetracordo grave hacia dugah.

Letras

Me kemi y me inflami

Cuando te vide yo a ti

El sentirte a ti cantar

Me pareces un bilbil

Ven a mi lado

Ven que to rogo

Ven mas presto tu biju

Que vo salir loco

Tus ojos que me miran

El corason me queman

Con estas miradas tuyas

Cuantas almas quemaron

Ven a mi lado...

\section{2. "No Riyas con mi»}

Número: 3001-B

Artista: Hazan, Victoria

Otros artistas: letras de Victoria Hazan. Música de Nick D(oneff), violín. Oriental Trío (Doneff; Melko Melkon, oud; Kanuni Garbis [Bakirgian], kanun)

Matrix: C.V.-3001-B

Grabado: 1942 en NY

23 Ese sexto grado rebajado se llama acem. Ese comportamiento es muy común en todos los modos que usan la escala diatónica. Se denomina comportamiento diatónico. 


\section{Análisis musical}

\section{Makam: rast}

Escala: diatónica (Fig. 2)

Base: rast

Notas importantes: rast, segah, neva

Idiomatismos: una de las modulaciones típicas en este makam ${ }^{24}$ es la de tetracordo Huzzam a partir de la nota neva. En esta pieza encontramos este fenómeno muy desarrollado, tanto que se puede pensar que la pieza entera está en el makam Huzzam o Segah. Pero las terminaciones claras hacia la base muestran su verdadera naturaleza. Otra peculiaridad es que la nota neva se usa tanto para construir un tetracordo Huzzam como un tetracordo Ussak cambiando constantemente la afinación de las notas. En este segundo caso del uso del neva, cuando la melodía supera el ámbito de la octava, el segah agudo se presenta bastante rebajado porque ya su función es que sea una sexta menor ${ }^{25}$.

Desarrollo: ascendente con especial matización en la región aguda del modo. La grabación contiene una pequeña improvisación vocal (gazel), en el mismo makam.

\section{Letras}

No riyas con mi con ti me aravie

Tu profundo amor nunca lo aprecie

Que va ser de mi que vo a devinir

Del amor por ti yo vo tresalir

gazel

Ya no puedo mas cantar querida

Ni mis ojos mas yorar

Esto perdiendo la mi vida

Sin poder alcanzar

Agora creyi tu verdadero amor

Agora supe cualo es el amor

24 Véase también en línea: https://www.neyzen.com/makamlar/rast.html. Una muy interesante página que contiene repertorio desde el siglo XIV hasta contemporáneo.

25 En este caso se cumple la regla del comportamiento diatónico. 
5.3. «Un $A \tilde{n} o$ ay» ${ }^{26}$

Número: 3002-A

Artista: Hazan, Victoria

Otros artistas: letras de Victoria Hazan. Música de Nick D(oneff), violín. Oriental Trío (Doneff; Melko Melkon, oud; Kanuni Garbis [Bakirgian], kanun)

Matrix: CV-3002-A

Grabado: 1942 en NY

\section{Análisis musical}

Makam: Hicaz

Escala: cromática (Fig. 7)

Base: dugab

Notas importantes: dugah, neva

Idiomatismos: este makam desarrolla el tetracordo cromático tanto en la región grave como en la aguda y por esa razón la forma de la escala es simétrica. Muy común también en este repertorio es terminar con la melodía en la octava aguda.

\section{Letras}

Un año ay que te esto esperando

Ainda tu no vienes

Tu me enganiates

Tu me rivinates

Del dio que lo topes

Cayi tanto desolado

No bay quien demande por mi

Ten piedad non me mates

Ven cuando te yamo

\section{4. "Mis Penserios me Trucheron»}

Número: 3002-B

Artista: Hazan, Victoria

26 En línea: http://www.panossavopoulos.gr/p/6.html 
Otros artistas: letra de Victoria Hazan. Música de Nick D(oneff), violín. Oriental Trío (Doneff; Melko Melkon, oud; Kanuni Garbis [Bakirgian], kanun)

Matrix: CV-3002-B

Grabado: 1942 NY

\section{Análisis musical \\ Makam: ussak \\ Base: dugab \\ Escala: diatónica \\ Entrada: en el neva}

Notas importantes: dugah, neva, huseyni

Idiomatismos: en el gazel, al ser una forma libre de interpretación, el cantante adapta el texto que, la mayoría de las veces, inventa en el momento. Aun así, dado que las reglas para seguir el desarrollo son las mismas en cada cantante, una vez que se ha de improvisar sobre un mismo makam (pues los movimientos melódicos conducen hacia los mismos grados importantes), podemos encontrar melodías parecidas en distintos gazel dentro de un mismo modo. Esto sucede entre las estrofas, o más bien cuando se realiza el primer descenso vocal, ya que un instrumentista que domina bien el lenguaje improvisa en modo de imitación o desarrollando ideas de las que la voz ha presentado anteriormente. De este modo, se acaba de configurar una melodía rítmica y alegre.

Desarrollo: normalmente el desarrollo melódico de esta forma se divide en dos partes. En la primera se realiza una entrada en la región grave del modo y en la segunda en el resto de los sonidos de la escala.

\section{Letras}

Ob mis penserios me trucheron

Una grande cayades

Por una morena dulce

Mi vida va depiedrer aman, yarei, aman ei

Ob Aman dame un cavello de tu cavesa

Los ojos me cusire aman

Jurare delante del dio santo

Que otra de ti non amare 


\section{5. «Un Dia yo Bezi»}

Número: 3003-A

Artista: Hazan, Victoria

Otros artistas: letras de Victoria Hazan. Música de Nick D(oneff), violín. Oriental Trío (Doneff; Melko Melkon, oud; Kanuni Garbis [Bakirgian], kanun)

Matrix: C.V.-3003-A

Grabado: 1942 en NY

\section{Análisis musical}

Makam: neveser y nibavent

Base: rast

Notas importantes: rast, neva, buseyni

Escala: mixta partiendo de la nota rast, diatónica y cromática (Fig. 4)

Idiomatismos: a tiempo de vals con un contexto melódico más cercano al modo menor, esta pieza usa distintos tetracordos los cuales la cantante utiliza libremente.

\section{Letras}

Un dia yo bezi tu cara hermosa

Cuando estabas durmiendo

Me parecio que es una rosa

Que estaba goliendo

Ob liinda rosa de primavera

Que se avriyo en goliendo

Era tu cara muy hermosa

Que estava goliendo

\section{6. "Todas mis Esperanzas»}

Número: 3003-A

\section{Artista: Hazan, Victoria}

Otros artistas: letras de Victoria Hazan. Música de Nick D(oneff), violín. Oriental Trío (Doneff; Melko Melkon, oud; Kanuni Garbis [Bakirgian], kanun) Matrix: C.V.-3003-A 
Grabado: 1942 en NY

Análisis musical

Makam: huzzam

Base: segab

Notas importantes: segah, neva

Escala: cromática suave (Fig. 5)

Idiomatismos: es una forma de interpretación muy peculiar. Hazan usa una canción turca de Yesârî Asım Arsoy, «Umitlerim hep kirildi» ${ }^{27}$, en la cual adapta el texto a la primera parte de la canción. Como podemos observar usa las notas importantes de la melodía para construir las cadencias y así expresar el texto en ladino. La canción sigue después de esa introducción libre con su melodía original, con ritmo y con los versos en ladino.

\section{Letras}

Todas mis esperanzas

Todas fueron en vano

A mi lado non vas a venir

Lagrimas de los mis ojos

Me callen por tus amores

Un día yo non alegri

Ni tu tambien no vas alegrar

Si me esparto y si yoro

De este amor nunca me ulvido

\section{7. «Lagrimas Vertere»}

Número: 3004-A

Artista: Hazan, Victoria

Otros artistas: letras de Victoria Hazan. Música de Nick D(oneff), violín. Oriental Trío (Doneff; Melko Melkon, oud; Kanuni Garbis [Bakirgian], kanun)

Matrix: C.V.-3004-A

27 Véase en línea: https://www.neyzen.com/nota_arsivi/02_klasik_eserler/046_huzzam/ umitlerim_hep_kirildi.pdf 
Grabado: 1942 en NY

\section{Análisis musical}

Makam: rast

Escala: diatónica

Base: rast

Notas importantes: rast, segah, dugah, cargah

Idiomatismos: como la canción anterior, es una libre adaptación del texto a la canción turca de Mehmet Baha Pars «Her yer karanlik» ${ }^{28}$. Es muy interesante el hecho de usar las notas importantes para construir las melodías intermedias, lo cual ayuda a la construcción de la pieza y al desarrollo de los melismas.

\section{Letras}

Lagrimas vertere sin poderlas ver

Ob dio asme saver la mi vida que va ser

Esclamar esclami de dolores me inchi

Entera yo me atemi

En la red que yo cayi

Una tomba yo fragui

Para ti y para mi

Es la tomba la primera

que nos hace despartir

\section{8. «Seda amarilla son tus Cavellos»}

Número: 3004-B

Artista: Hazan, Victoria

Otros artistas: letras de Victoria Hazan. Música de Nick D(oneff), violín. Oriental Trío (Doneff; Melko Melkon, oud; Kanuni Garbis [Bakirgian], kanun)

Matrix: C.V.-3004-B

Grabado: 1942 en NY

28 Véase en línea: https://www.neyzen.com/nota_arsivi/02_klasik_eserler/075_rast/ her_yer_karanlik.pdf 


\section{Análisis musical}

Makam: huzzam

Escala: cromática suave

Base: segab

Notas importantes: segah, neva, evic

Desarrollo: la escala tiene dos polos: el tetracordo Huzzam grave con base la nota segah y el tetracordo Huzzam agudo con base en la nota evic. Como es común, primero se desarrolla la parte grave y después la parte aguda y en este caso el estribillo sirve para la vuelta.

\section{Letras}

Seda amarilla son los tus cavellos

Con mis manos entrensados

De mis ojos callen lagrimas

Por mi yero que cometi

Tu con migo abrasarnos

Y presiar tus entrensados

Vente con mi tu mi biju

Te amare $y$ te adorare

\section{9. «El Cante por la Victoria»}

Número: 3005-A

Artista: Hazan, Victoria

Otros artistas: letras de Victoria Hazan. Música de Nick D(oneff), violín. Oriental Trío (Doneff; Melko Melkon, oud; Kanuni Garbis [Bakirgian], kanun)

Matrix: C.V.-3005-A

Grabado: 1942 en NY

\section{Análisis musical}

Makam: Acem kurdi

Base: dugab

Escala: diatónica variada (Fig. 3)

Notas importantes: dugah, neva, acem 
Desarrollo: las entradas se hacen en la nota neva enfatizando siempre también la nota acem. Pero las frases siempre reposan en $d u g a h$.

Idiomatismos: otra canción relacionada con un repertorio común tocado y disfrutado en las tabernas. Esta vez relacionado con el rebetiko, género musical muy popular en Grecia hasta hoy en día. La introducción de la canción está grabada unos años antes (1936) con el título: «Fora ta Mavra fora ta» ${ }^{29}$ (Фó $\rho \alpha \tau \alpha$ $\mu \alpha v ́ \rho \alpha$

Letras

De mi jente me esparti

A la armada me enlisti

Un año de anio de amor pasi con ti querida

Por fin de ti yo me aleshi

Non yores tu mi querida

Bivo y sano vo a vuelver

Tengo en el dio la esperanza

Que la victoria vamos tener

Uncle Sam ya mos yamo

Con mucho bueno el mos trato

Tengo en el dio la esperanza

De Hitler tomaremos la venganza

Ijos queridos aunarvos

$Y$ del peligro salvarnos

Con vuestra fuersa queridos ijos

Salvación a la nacion

29 Fuente en línea: https://www.youtube.com/watch?v=Mmmd2gSz8wE 
5.10. "No bay Luz en mis Ojos»

Número: 3005-B

Artista: Hazan, Victoria

Otros artistas: letras de Victoria Hazan. Música de Nick D(oneff), violín. Oriental Trío (Doneff; Melko Melkon, oud; Kanuni Garbis [Bakirgian], kanun)

Matrix: C.V.-3005-B

Grabado: 1942 en NY

\section{Análisis musical}

Makam: Sabah

Base: dugah

Escala: mixta diatónica y cromática (Fig. 6)

Notas importantes: dugah, cargah, buseyni

Desarrollo: dos polos de atracción: tetracordo Sabab en la región grave y tetracordo Hicaz en la aguda.

Idiomatismos: característico sonido el del cuarto grado rebajado para crear la atracción hacia el tercer grado usándolo con frecuencia como centro importante. Es un makam muy usado para la improvisación vocal y con frecuencia se expresa con palabras de mucha pena. Características las sílabas yarei, aman, ei como terminaciones de melismas difíciles y expresión de pena y dolor.

Letras

Ob! No bay luz en mis ojos

Ni claridad en mi corason

Ob! Se escurecio toda mi vida

Por tenerte mucho amor yarei, aman ei

Ob! Con mi boca me encapi querida

Que palabra a ti te di

$\mathrm{Ob!}$ ! Me ensendites en un fuego

Sin poderme consintir (2)

Yarei aman ei... 
CHRISTOS KANELLOS MALAMAS

VICTORIA HAZAN Y SUS GRABACIONES DE MÚSICA SEFARDÍ EN NY (1942)

\section{CONCLUSIONES}

Nos hemos acercado a un repertorio muy especial de música sefardí, relacionado con la música turca de tradición popular, para intentar proponer un análisis adecuado y propio para este género, el cual nos puede permitir comprender estas sonoridades muy usadas en un repertorio musical originario de las tabernas de Esmirna. Somos conscientes de que sin la experimentación y el acercamiento práctico a las piezas y sin conocer el funcionamiento del sistema del makam turco es prácticamente imposible llegar a interpretarlas. Al mismo tiempo, hemos visto la riqueza melódica e interválica que constituye esta música y que es posible adaptar las letras a las melodías preexistentes conduciendo el fraseo melódico hacia las notas importantes. También hemos observado la importancia de la improvisación en la interpretación.

Nos hemos limitado a comentar muy pocos aspectos sobre la modalidad oriental, notaciones que se pueden usar y todo tipo de cuestiones teóricas que ayudarían al lector a entender mejor las piezas. Pero nuestro fin es crear un primer contacto con la materia y no un manual de música turca, que esperemos que pronto vea la luz. Sobre todo, nuestro propósito ha sido hacer ver esas músicas en su contexto modal y según ese contexto familiarizarnos con los sonidos y sobre todo con la interválica usada.

\section{BIBLIOGRAFÍA}

Bali, N. R. (2013). From Anatolia to the New World Life Stories of the First Turkish Immigrants to America. Estambul.

Bucuvalas, T. (2018). Greek Music in America. University Press of Mississipi.

Díaz-Mas, P. (2009). Folk Literature among Sephardic Bourgeois Women at the Beginning of the Twentieth Century. European Journal of Jewish Study, 3.1, 81-101.

Díaz-Mas, P. y Sánchez Pérez, M. (2012). La comunidad sefardí de Los Ángeles (California) y su periódico El Mesajero/The Messenger. eHumanista, 37, 153-171.

Havassy Rivca, S. E. (2020). Eastern Mediterranean Judeo-Spanish Songs from the EMI Archive Trust (1907-1912). En Anthology of Music Traditions in Israel, 27. Jerusalem.

López Núñez, M. T. y Sánchez Sánchez, A. M. (2017). Irene y Alice Lewishon. Dos mujeres pioneras en la representación músico-teatral al servicio del Trabajo Social. ArtyHam Revista de Artes y Humanidades, 37, 108-164.

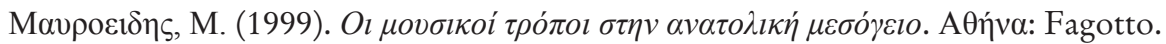

Signell, L. K. (1977). Makam, modal practice in Turkish art music. Asian Music Publications. 


\section{WEBGRAFÍA}

https://www.sephardicmusic.org/artists/Hazan,Victoria/Hazan,Victoria.htm https://jewishstudies.washington.edu/bailar-a-la-turka-78-rpm-records-in-seattle-sepharadi-households/why-baruchs-and-friends-play-turkish-records/

\section{ANEXOS}

Tabla 1. Las canciones, clasificación por modos y formas

\begin{tabular}{|c|c|c|c|}
\hline $\begin{array}{l}\text { Canción } \\
\text { sefardí }\end{array}$ & Forma & Modo & Procedencias, similitudes \\
\hline $\begin{array}{l}\text { «El Cante por } \\
\text { la Victoria» }\end{array}$ & Şarkı & $\begin{array}{l}\text { Acem } \\
\text { kurdi }\end{array}$ & 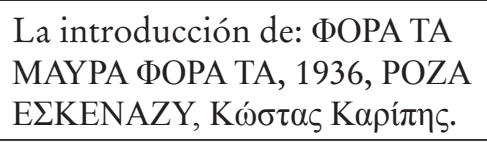 \\
\hline $\begin{array}{l}\text { «Lagrimas } \\
\text { Vertere» }\end{array}$ & $\begin{array}{l}\text { Şarkı, libre } \\
\text { interpretación }\end{array}$ & Rast & $\begin{array}{l}\text { Mehmet Baha Pars: «Her yer } \\
\text { karanlik» }\end{array}$ \\
\hline $\begin{array}{l}\text { «Me Kemi y } \\
\text { me Enflami» }\end{array}$ & Şarki & $\begin{array}{l}\text { Huseyni, } \\
\text { kurdi }\end{array}$ & \\
\hline $\begin{array}{l}\text { «Mis } \\
\text { Penserios» }\end{array}$ & $\begin{array}{l}\text { Gazel, } \\
\text { improvisación } \\
\text { libre }\end{array}$ & Kurdi & \\
\hline «No Ay Luz» & $\begin{array}{l}\text { Gazel, } \\
\text { improvisación } \\
\text { libre }\end{array}$ & Sabab & \\
\hline $\begin{array}{l}\text { «No Riygas } \\
\text { con mi» }\end{array}$ & Şarkı & $\begin{array}{l}\text { Rast, } \\
\text { Huzzam }\end{array}$ & \\
\hline $\begin{array}{l}\text { «Seda amarilla } \\
\text { son tus } \\
\text { Cavellos» }\end{array}$ & Şarkı & Huzzam & \\
\hline $\begin{array}{l}\text { «Todas mis } \\
\text { Esperansas» }\end{array}$ & $\begin{array}{l}\text { Şarkı, libre } \\
\text { interpretación }\end{array}$ & Huzzam & $\begin{array}{l}\text { Yesârî Asım Arsoy: «Umitlerim } \\
\text { hep kirildi» }\end{array}$ \\
\hline $\begin{array}{l}\text { «Un Anio } \\
\text { Ay» }\end{array}$ & Şarkı & Hicaz & \\
\hline $\begin{array}{l}\text { «Un Dia yo } \\
\text { Bizi» }\end{array}$ & Şarkı & $\begin{array}{l}\text { Neveser, } \\
\text { Nibavent }\end{array}$ & \\
\hline
\end{tabular}



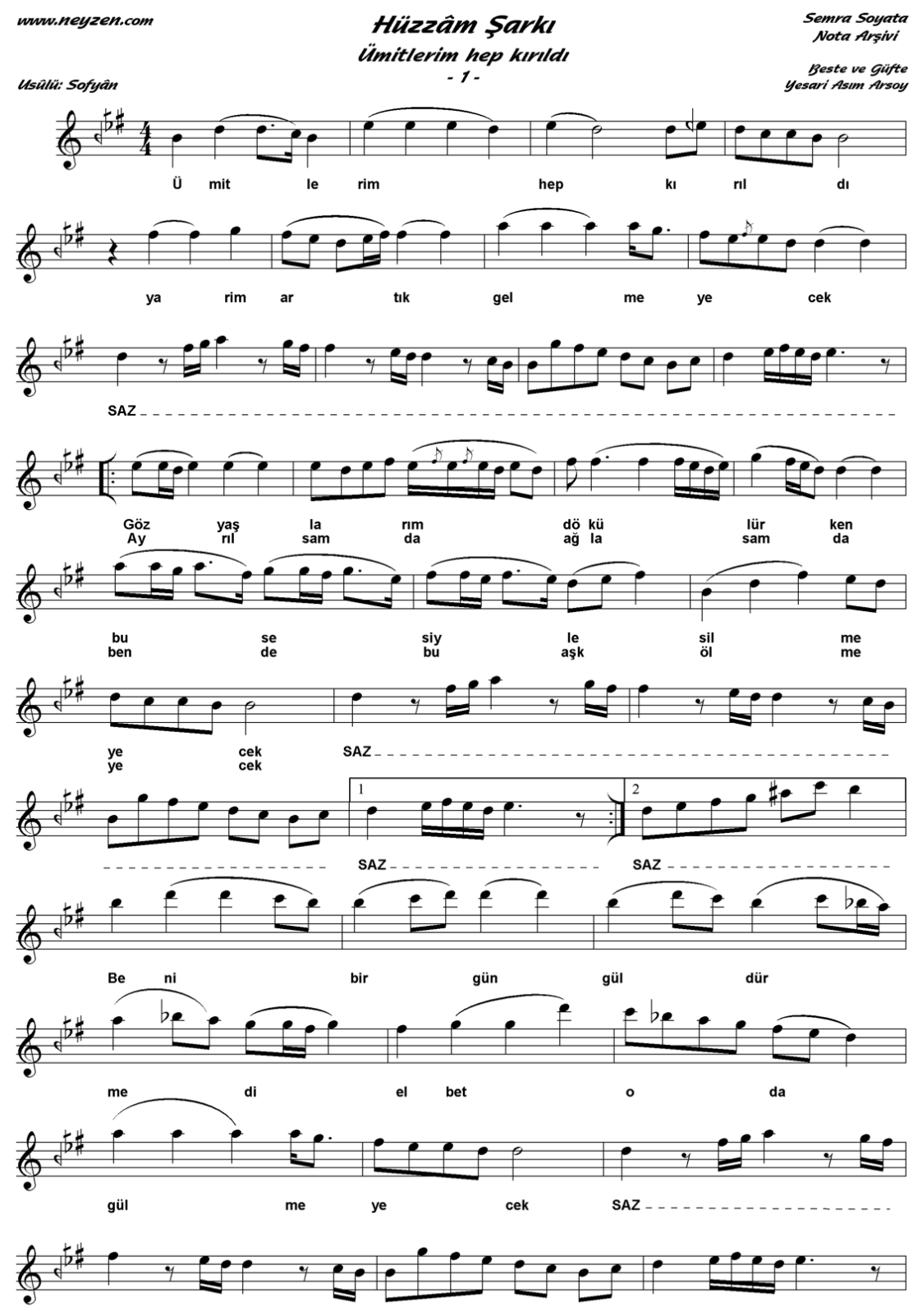

Figura 8. La canción original de «Lagrimas Vertere».

Ediciones Universidad de Salamanca / 요

Popular Music Research Today, 3, 2 (2021), pp. 41-67 

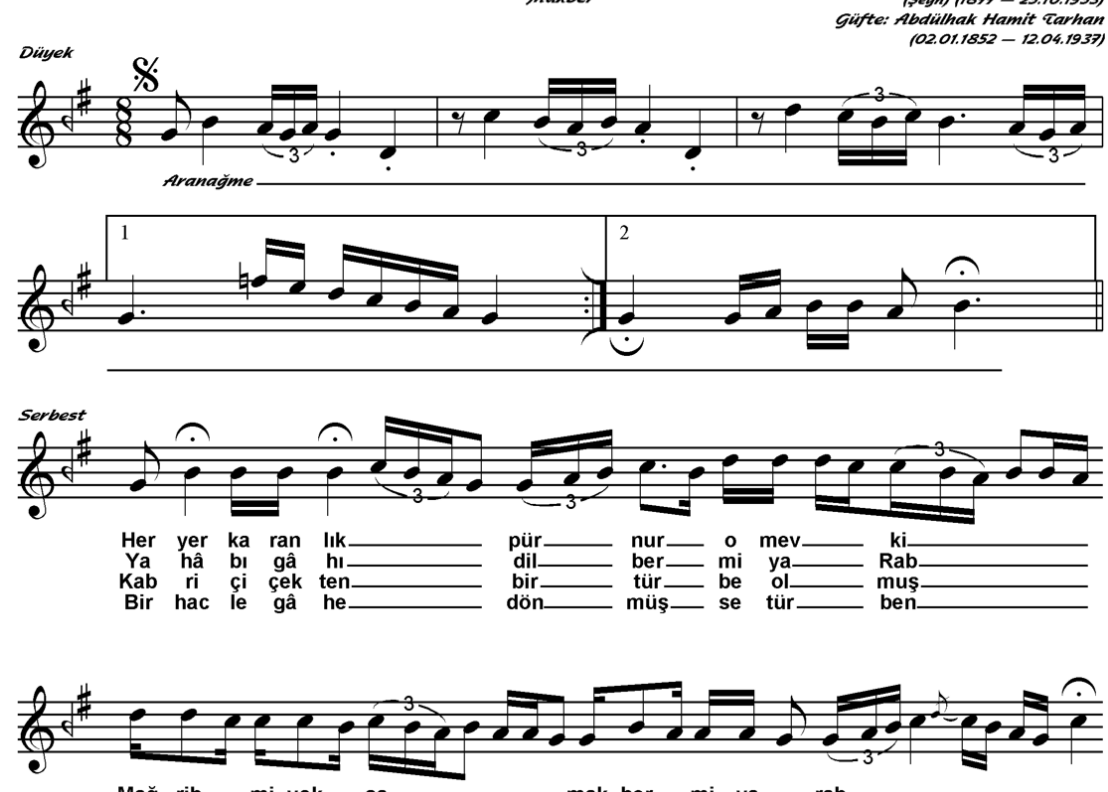

Mağ rib_ mi yok_ sa___ mak ber_ mi ya__ rab

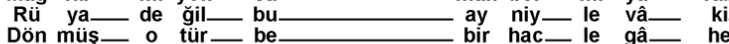

Aç koy_ nu nu_ aç_ mâ şu_ ku num_ ben
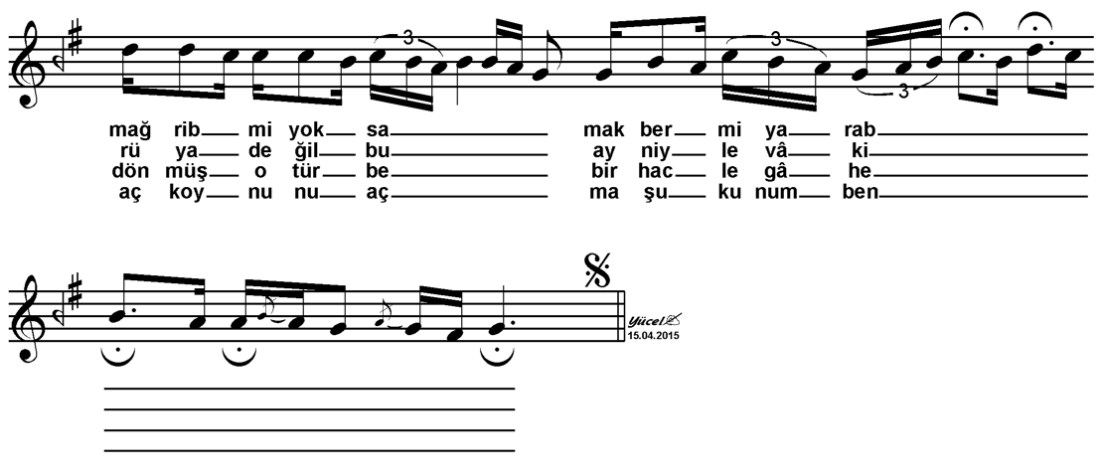

Her yer karanlık pür-nûr o mevkî Mağrib mi yoksa makber mi yâ Râb Yầ hâbgâh-1 dilber mi yâ Rẩ Rüyâ değil bu ayniyle vakî.
Kabrin ciçekten bir türbe olmus Dönmüss o türbe bir haclegâhe Bir haclegâhe dönmüssse türben Aç koynunu aç maşukânim ben.

Figura 9. La canción original de «Todas mis Esperanzas».

Ediciones Universidad de Salamanca / @D@ 


\section{El cante por la victoria}

Transcripción: Christos Kanellos Malamás
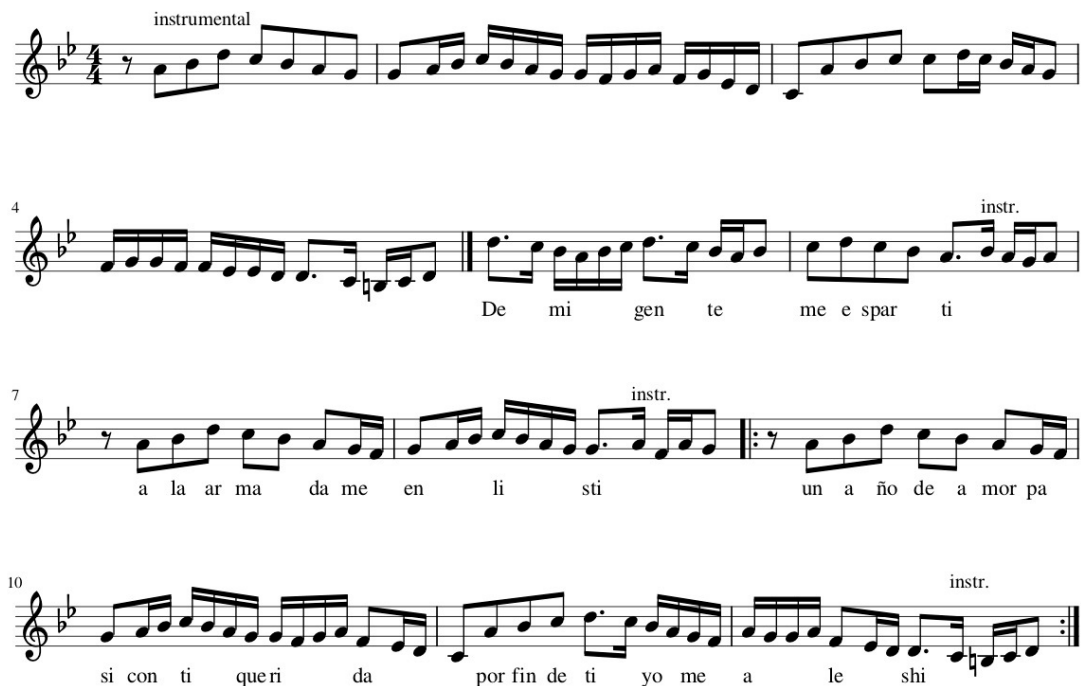

Figura 10. «El Cante por la Victoria» (transcripción del autor).

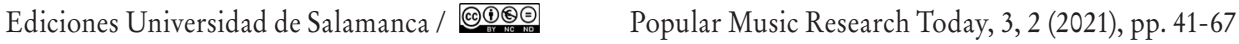




\section{Me kemí y me enflamí}

Transcripción: Christos Kanellos Malamás
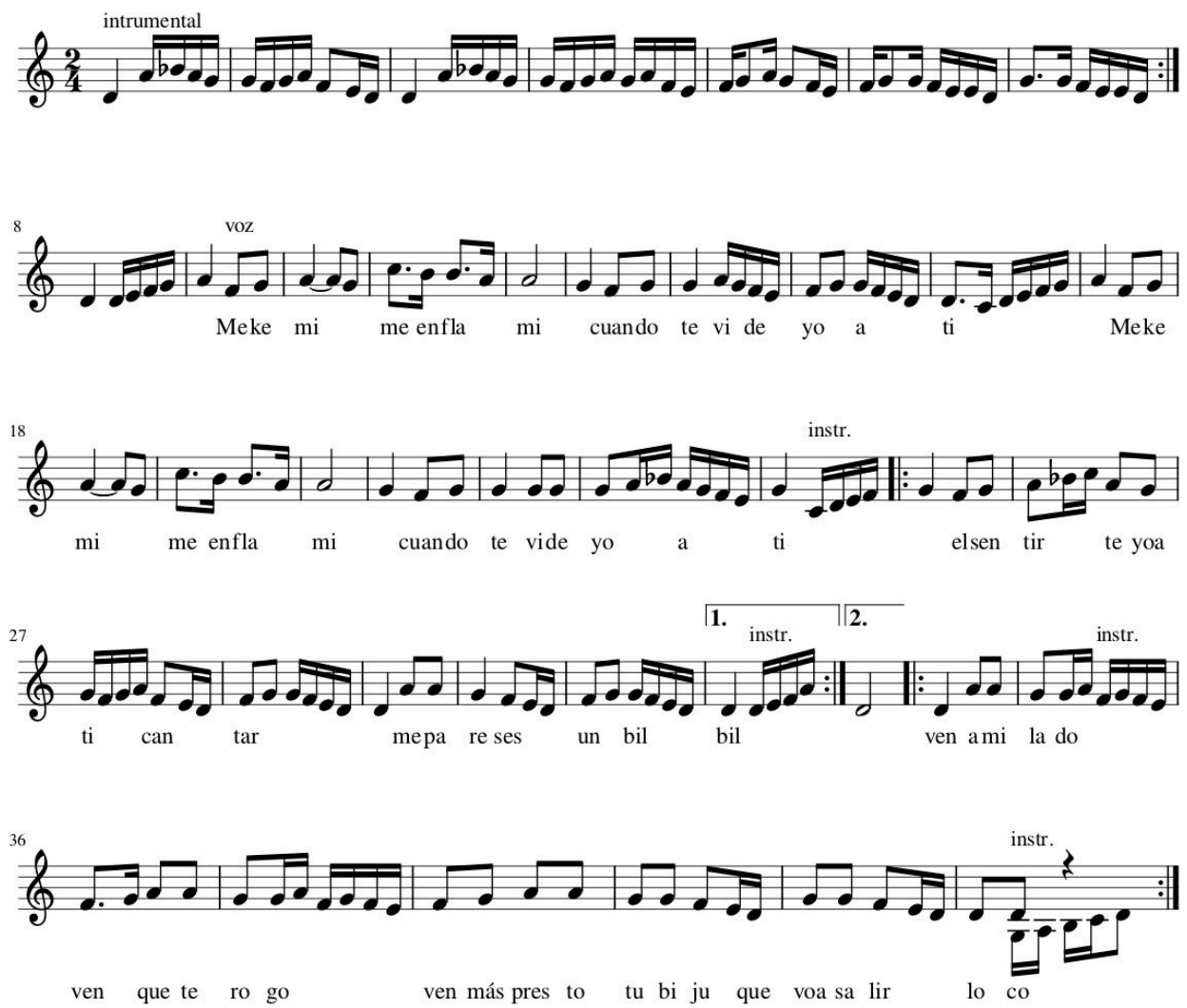

Figura 11. «Me Kemi y me Enflami» (transcripción del autor).

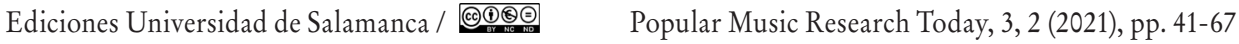


